\title{
Phase Slip in Charge-Density-Wave Systems
}

\author{
Satish Ramakrishna, ${ }^{(1)}$ Michael P. Maher, ${ }^{(1)}$ Vinay Ambegaokar, ${ }^{(1)}$ and Ulrich Eckern ${ }^{(2)}$ \\ ${ }^{(1)}$ Laboratory of Atomic and Solid State Physics and Materials Science Center, Cornell University, Ithaca, New York 14853 \\ ${ }^{(2)}$ Kernforschungszentrum Karlsruhe, Institut für Nukleare Festkörperphysik, Postfach 3640, Karlsruhe D7500, Germany
}

(Received 23 October 1991)

\begin{abstract}
Recent experiments in charge-density-wave systems suggest that thermally activated phase slip is responsible for current flow. We present a quantitative theory that explains the nonlinear current-voltage relationship.
\end{abstract}

PACS numbers: 71.45. Lr, 72.15. Nj

The currently accepted model for charge-density-wave (CDW) dynamics gives the phase of the CDW order parameter the leading role in determining the dynamics far below the Peierls transition temperature. This model has been successful in explaining many important transport properties: the existence of a threshold electric field for conduction, extremely non-Ohmic conductivity, narrowband noise, mode locking [1], etc.

The "phase only" model cannot, however, suffice at current contacts, where normal current is converted into CDW (also called Fröhlich) current. To one side of the contact, the CDW is moving [2], while on the other side, the CDW and its wave fronts are stationary. If CDW wave fronts were not removed continually at one contact and injected at the other, a large phase difference would build up very quickly across each one and this would destroy the condensed state. In short, the CDW must nucleate topological dislocations that carry away the extra phase difference: We refer to this process as phase slip. This process is best studied [3] in the "transposed" configuration (Fig. 1). In the usual four-probe "normal" configuration, a current $I$ is injected at the sample's ends
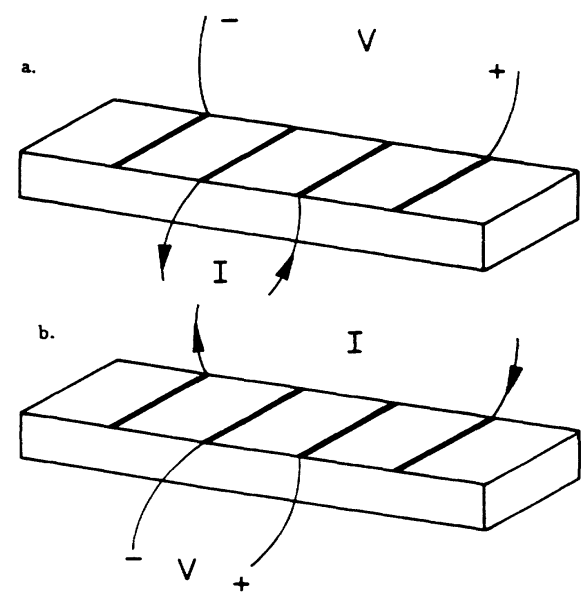

FIG. 1. (a) Transposed and (b) normal configurations. and the voltage is measured by contacts placed a distance $L$ apart. This configuration separates the regions of current conversion and voltage measurement. In the transposed configuration, however, it is the current contacts that are placed a distance $L$ apart, between the voltage contacts. In the normal configuration, the voltage developed across a section of length $L$ is $V_{n}=\mathscr{E}_{T} L$ $+I_{\mathrm{CDW}} r_{\mathrm{CDW}} L$, where $\mathscr{E}_{T}$ is the usual bulk threshold field, $I_{\mathrm{CDW}}$ is the CDW current, and $r_{\mathrm{CDW}}$ the resistance per unit length. Recent experiments [3-6] on phase slip at contacts found that there is a difference between the voltage across a length $L$ in the transposed and normal configurations when the same current $I_{\mathrm{CDW}}$ flows. We may write the voltage required in the transposed configuration as $V_{t}=V_{n}+V_{\mathrm{ps}}$, where $V_{\mathrm{ps}}$, the difference between the voltages in the two configurations, is referred to as the phase-slip voltage. In the above, the normal carriers have not been neglected; they are a parallel channel of conduction and they experience the same potential difference as the CDW.

The aim of this paper is to establish that $V_{\mathrm{ps}}$ drives the nucleation of phase-slip centers between the current contacts. This extra voltage cannot appear only at the current contacts, over some small length. If it did, there would be a huge current of normal carriers due to the large electric field. Such large normal currents have not been observed in the experiments. It is, additionally, not possible for the electric field to be discontinuous in a small region in a linear sample with homogeneous electrical conductivity. We are thus led to a picture where the voltage appears throughout the sample, across the current contacts, with the consequence that a proportionate amount should be seen in the normal configuration. It is very small and would be hard to observe in that configuration unless the voltage contacts were almost as widely spread as the current contacts.

To relate phase slip to the CDW current, we note that in the steady state the rate at which the CDW wave fronts are lost at the current contact must equal the rate of phase slip. What follows in the paper is a computation of this phase-slip rate, carried out using the usual Ginzburg-Landau static free energy [7] $\mathcal{F}$ : 


$$
\begin{aligned}
\mathscr{F}\left[\Delta e^{i \phi}\right]= & \int d^{3} r \frac{1}{2} \Delta^{2}\left[K_{x}\left(\frac{\partial \phi}{\partial x}\right)^{2}+K_{y}\left(\frac{\partial \phi}{\partial y}\right)^{2}+K_{z}\left(\frac{\partial \phi}{\partial z}\right)^{2}\right] \\
& +\frac{1}{2}\left[K_{x}\left(\frac{\partial \Delta}{\partial x}\right)^{2}+K_{y}\left(\frac{\partial \Delta}{\partial y}\right)^{2}+K_{z}\left(\frac{\partial \Delta}{\partial z}\right)^{2}\right]-\frac{1}{2} \alpha \Delta^{2}+\frac{1}{4} \beta \Delta^{4}-\frac{e \rho_{s}(\Delta)}{Q} \mathscr{E} \phi+V_{\text {imp }}(\phi) .
\end{aligned}
$$

We shall take the CDW axis to be along the $z$ direction. The $K$ 's are the anisotropic elastic constants, $\rho_{s}$ is the appropriate condensate density [8] (we use the static limit for low currents and the dynamic limit for large currents corresponding to electric fields much above threshold), $\mathscr{E}$ is the applied electric field, $e$ is the absolute value of the electron's charge, $Q=2 k_{F}$, and $V_{\text {imp }}(\phi)$ is the impurity potential.

The samples used must be considered three dimensional, since the transverse amplitude coherence lengths $\left(\xi_{A}^{T}\right)$ are $\sim 10 \AA$ and are much smaller than the typical transverse sample dimensions (which are $\sim 1 \mu \mathrm{m}$ ). The simplest intensive (i.e., independent of the sample dimensions) process one can imagine to remove or add a wave front is the creation of an edge dislocation loop, i.e., oriented with the plane of the loop perpendicular to the CDW direction $[9,10]$.

To understand the mechanics of dislocation loop nucleation, we view the CDW as an elastic medium [9] with a displacement field $\mathbf{u}=-\mathbf{e}_{z} \phi / Q$ and strain tensor components

$$
\begin{aligned}
& \epsilon_{z z}=-\frac{1}{Q} \frac{\partial \phi}{\partial z}, \quad \epsilon_{x z}=\epsilon_{z x}=-\frac{1}{Q} \frac{\partial \phi}{\partial x}, \\
& \epsilon_{y z}=\epsilon_{z y}=-\frac{1}{Q} \frac{\partial \phi}{\partial y} .
\end{aligned}
$$

The structure of the elastic terms in (1) allows us to write the components of the stress tensor as

$$
\begin{aligned}
& \tau_{z z}=K_{z} \Delta^{2} Q^{2} \epsilon_{z z}, \quad \tau_{x z}=\tau_{z x}=K_{x} \Delta^{2} Q^{2} \epsilon_{x z}, \\
& \tau_{y z}=\tau_{z y}=K_{y} \Delta^{2} Q^{2} \epsilon_{y z} .
\end{aligned}
$$

By separating the phase into background and dislocation [11] parts, we establish that it is background stresses that drive dislocation loop nucleation. In fact, only $\tau_{z z}$ (denoted by $\Sigma$ ) is relevant for the nucleation of edge loops.

Suppose an edge dislocation loop is created in a background stress $\Sigma$. The stress produces a radially outward force, the Peach-Koehler [12] force, upon the loop perimeter. In vector notation, the force per unit length is $\mathbf{F}=\boldsymbol{\Sigma} \times \boldsymbol{\kappa} / Q$, where $\boldsymbol{\kappa}$ is the vorticity. The outward force on an edge loop of vorticity $2 \pi$ is hence $4 \pi^{2} \Sigma R / Q$, where $R$ is the loop radius. The energy gain when the loop radius is increased from 0 to $R$ is then $2 \pi^{2} R^{2} \Sigma / Q$. On the other hand, the curvature energy [13] of a dislocation loop is $2 \bar{K} \Delta^{2} \pi^{2} R\left(\ln \left[8 R / \xi_{A}^{T}\right]-\frac{7}{4}\right)$, where $\bar{K}$ is an effective transverse elasticity [14]. Thus, as in all such nucleation problems, vortices that are below a critical size just fall back upon themselves, while those bigger than this size grow indefinitely. The nucleation energy of the critical dislocation loop is

$$
\delta \mathfrak{F}=e \frac{\pi^{2}}{2} \frac{\left(\bar{K} \Delta^{2}\right)^{2} Q}{\Sigma},
$$

where

$$
\mathcal{C}=\left(\ln \left[\frac{8 R_{C}}{\xi_{A}^{T}}\right]-\frac{3}{4}\right)\left(\ln \left[\frac{8 R_{C}}{\xi_{A}^{T}}\right]-\frac{11}{4}\right)
$$

and is a dimensionless factor of order unity in the regions of importance to the experiment. We note that the nucleation energy is inversely proportional to $\Sigma$.

We have yet to compute the background stress $\Sigma$. We now demonstrate that it is related to the phase-slip voltage. The effect of the current contacts (separated by a distance $L$ ) is accounted for in the phase $\phi$ by writing $\phi=\phi_{0}+\phi_{1}$, where $\phi_{0}$ represents the bulk solution to the CDW problem and $\phi_{1}$ is a small time-independent correction. We then expand the usual overdamped equation of motion [15] (for $\phi$ ) for small $\phi_{1}$ (we average over transverse dimensions since phase coherence is maintained along those directions):

$$
\begin{aligned}
\gamma \dot{\phi}_{0}-K_{z} \Delta^{2} \frac{\partial^{2} \phi_{0}}{\partial z^{2}}- & K_{z} \Delta^{2} \frac{\partial^{2} \phi_{1}}{\partial z^{2}} \\
& =\frac{e \rho_{s}}{Q} \mathscr{E}+F_{\mathrm{imp}}\left(\phi_{0}\right)+\frac{\partial F_{\mathrm{imp}}}{\partial \phi} \phi_{1}
\end{aligned}
$$

We are interested in time-independent effects that vary slowly in space. Hence, upon canceling out the terms that arise from the averaged bulk equation [16], noting that $V_{\text {imp }}(\phi)$ is simply periodic in $\phi$, and defining $\mathscr{E}_{\mathrm{ps}}$ $=V_{\mathrm{ps}} / L$, we are led to

$$
K_{z} \Delta^{2} \frac{\partial^{2} \phi_{1}}{\partial z^{2}}=-\frac{e \rho_{s}}{Q} \mathscr{E}_{\mathrm{ps}}-\phi_{1} V_{\mathrm{imp}}(\phi) .
$$

The factor multiplying $\phi_{1}$ in the above equation is the pinning energy density above threshold. This quantity decreases rapidly [17] above threshold and we assume it to be negligible. We are then left with

$$
K_{z} \Delta^{2} \frac{\partial^{2} \phi_{1}}{\partial z^{2}}=-\frac{e \rho_{s}}{Q} \mathscr{E}_{\mathrm{ps}} .
$$

By construction, $\phi_{1}$ obeys periodic boundary conditions; the solution to the above equation then is

$$
\begin{aligned}
K_{z} \Delta^{2} \phi_{1}(z)=- & \left.\frac{1}{2} \frac{e \rho_{s}}{Q} \mathscr{E}_{\mathrm{ps} z} z-L\right) \\
& \rightarrow \Sigma=-K_{z} \Delta^{2} Q \frac{\partial \phi_{1}}{\partial z}=\frac{1}{2} e \rho_{s} V_{\mathrm{ps}}\left(\frac{2 z}{L}-1\right)
\end{aligned}
$$


We next briefly address the question of the magnitude of the contact strain [18]. The typical phase strain from weak pinning is $\sim \pi$ over a length scale of the Fukuyama-Lee-Rice phase correlation length. This is at least 10-100 times less than the contact-related strain for experimentally relevant values of the phase-slip voltage $(\sim 1 \mathrm{mV})$. Hence $(8)$ is an exceedingly accurate picture of the phase portrait.

This parabolic phase profile, and the corresponding stress distribution, indicates that the system favors the thermal nucleation of extra windings in one-half of the sample and the loss of windings from the other half. The spatially nonconstant stress in the sample leads to a position-dependent loop nucleation rate. The rate of thermally activated phase slip in, say, the section $0-L / 2$ is

$$
\mathcal{R}=\frac{\omega}{2 \pi} \frac{\mathcal{A}}{\left(\xi_{A}^{T}\right)^{2}} \int_{0}^{L / 2} \frac{d z}{\xi_{A}^{L}} e^{-\delta \mathcal{F}(z) / k_{B} T} .
$$

Since the dislocation loop could be nucleated anywhere in the cross-sectional area $(\mathcal{A})$ of the sample and in any of $L / \xi_{A}^{L}$ independently fluctuating regions [19] in the sample ( $\xi_{A}^{L}$ is the longitudinal amplitude coherence length), we include the prefactor $\mathcal{A} /\left(\xi_{A}^{T}\right)^{2}$ and scale $d z$ by $\xi_{A}^{L}$ in the integral. In $(10), \omega$ is an appropriate attempt rate. We have nothing concrete to say about it except that it is probably on the same scale as other typical microscopic rates $\sim 10^{11} \mathrm{~s}^{-1}$. It is convenient to define

$$
V_{a}=e \pi^{2} \frac{Q}{e \rho_{s}} \frac{\left(\bar{K} \Delta^{2}\right)^{2}}{k_{B} T}
$$

such that

$$
\frac{\delta \mathcal{F}(z)}{k_{B} T}=\frac{V_{a}}{V_{\mathrm{ps}}}\left(\frac{2 z}{L}-1\right)^{-1} .
$$

With the above rate at hand, the current is

$$
I_{\mathrm{CDW}}=2 \pi \frac{e \rho_{s}}{Q} \mathcal{A} \mathcal{R}\left[\frac{V_{\mathrm{ps}}}{V_{a}}\right],
$$

since each phase-slip event adds or removes one wave front, i.e., $2 \pi$ of phase, over the entire cross section of the sample. We now compute this current as a function of the phase-slip voltage. The integral for the rate can be simplified [11] to the Stieltjes asymptotic series

$$
\begin{aligned}
\mathcal{R}\left[\frac{V_{\mathrm{ps}}}{V_{a}}\right]= & \frac{\omega}{2 \pi} \frac{\mathcal{A}}{\left(\xi_{A}^{T}\right)^{2}} \frac{L}{4 \xi_{A}^{L}} \frac{V_{\mathrm{ps}}}{V_{a}} e^{-V_{a} / V_{\mathrm{ps}}} \\
& \times\left[1-2 ! \frac{V_{\mathrm{ps}}}{V_{a}}+3 !\left(\frac{V_{\mathrm{ps}}}{V_{a}}\right)^{2}-\ldots\right] .
\end{aligned}
$$

The typical scale of $V_{\mathrm{ps}}$ is $\sim 1 \mathrm{mV}$ so that $V_{\mathrm{ps}} / V_{a} \ll 1$ at low temperatures. We next use (13) and define

$$
I_{0}(L)=\omega \frac{e \rho_{s}}{Q} \mathcal{A} \frac{\mathcal{A}}{\left(\xi_{A}^{T}\right)^{2}} \frac{L}{4 \xi_{A}^{L}}
$$

to obtain

$$
I_{\mathrm{CDW}}=I_{0}\left(\frac{V_{\mathrm{ps}}}{V_{a}}\right) e^{-V_{a} / V_{\mathrm{ps}}}\left(1-2 ! \frac{V_{\mathrm{ps}}}{V_{a}}+\cdots\right) .
$$

This is the central result of our paper. It is directly amenable to experimental check. We note that the $I-V$ curve turns on abruptly due to the presence of the exponential term in (14); this behavior has been misconstrued as evidence for a threshold phase-slip voltage [3,5]. The theory predicts that there is a small probability of phase slip at points other than the current contact. This leads to the rather peculiar result that the CDW current, for fixed phase-slip voltage, is proportional to the distance between the current contacts. We note that the precise length dependence is determined by the exact contact geometry: Here the contacts have been placed at the crystal ends.

To estimate the magnitude of the effect, we use lowtemperature values [14] for $\mathrm{NbSe}_{3}$ and use $e \approx 1$. Then using the condensate density [8] in the dynamic limit $\left(\rho_{s} \sim \Delta\right)$, appropriate to motion above threshold, we obtain [defining $\Delta_{0}=\Delta(T=0)$ ]

$$
\begin{aligned}
& V_{a} \approx 3200 \frac{\left(\Delta / \Delta_{0}\right)^{3}}{T} \mathrm{mV} \\
& I_{0}(L) \approx 10^{7}\left(\frac{L}{1 \mu \mathrm{m}}\right)\left(\frac{\mathcal{A}}{10 \mu \mathrm{m}^{2}}\right)^{2}\left(\frac{\Delta}{\Delta_{0}}\right)^{4} \mathrm{~A}
\end{aligned}
$$

If there exists a minimum current $\left(I_{\min }\right)$ that could be reliably measured then the "threshold voltage" $[3,5] V_{\mathrm{ps} 0}$ is defined from the equation $I_{\min }=I_{\mathrm{CDW}}\left(V_{\mathrm{ps} 0}\right)$. In fact, using (16),

$$
V_{\mathrm{ps} 0}=\frac{V_{a}}{\ln \left[I_{0}(L) / I_{\min }\right]-\ln \left[V_{a} / V_{\mathrm{ps} 0}\right]} .
$$

This threshold voltage would have the temperature dependence $V_{\mathrm{ps} 0} \sim\left(\Delta / \Delta_{0}\right)^{3} / T$ and would be in the scale of $\sim 0.1 \mathrm{mV}$. We leave detailed comparisons to experiment to a forthcoming paper [6].

In conclusion, we have presented a theory that clarifies the phenomenon of current-related phase slip in chargedensity-wave systems. The main result is a derivation of the relationship between the CDW current and the phase-slip voltage. Apart from an undetermined attempt rate, the theory provides explicit expressions for all other quantities. This theory differs from previous approaches [3] in that it deals with three-dimensional samples and also clearly explains the role of $V_{\mathrm{ps}}$. The phase-slip phenomenon is especially important at low temperatures, and should play a significant role in the study of CDW dynamics. It is of general relevance for related systems, e.g., spin-density waves. These, and other issues, will be discussed in a future publication [11].

We wish to acknowledge useful discussions with Professor R. Thorne and Professor J. P. Sethna and thank J. R. Morris for comments upon the manuscript. S.R. and 
V.A. are supported by the Materials Science Center at Cornell University through Grant No. DMR-8818558A02. M.P.M. acknowledges support from IBM.

[1] J. McCarten et al. (to be published); R. E. Thorne, Ph.D. thesis, University of Illinois, 1987 (unpublished).

[2] N. P. Ong, G. Verma, and K. Maki, Phys. Rev. Lett. 52, 663 (1984); N. P. Ong and K. Maki, Phys. Rev. B 32, 6582 (1985).

[3] J. C. Gill, J. Phys. C 19, 6589 (1986).

[4] D. V. Borodin, S. V. Zaitsev-Zotov, and F. Ya. Nad', Zh. Eksp. Teor. Fiz. 93, 1394 (1987) [Sov. Phys. JETP 66, 793 (1987)].

[5] M. Maher, J. McCarten, T. Adelman, and R. E. Thorne, J. Synth. Met. 43, 4031 (1990).

[6] M. Maher, T. L. Adelman, S. Ramakrishna, J. McCarten, and R. E. Thorne (unpublished).

[7] H. Fukuyama and P. A. Lee, Phys. Rev. B 17, 535 (1978)

[8] From microscopic theory, the static condensate density $\rho_{c}(T) / \rho_{c}(T=0)=1-Y(T) \approx 0.65\left(\Delta / \Delta_{0}\right)^{2}$ while the dynamic condensate density $\rho_{s}(T) / \rho_{s}(T=0) \approx \pi \Delta / 4 T_{P}$ close to the Peierls temperature. However, the experimental (narrow-band noise) results are pretty well fitted by taking $\rho_{s}(T) / \rho_{s}(T=0) \approx \Delta / \Delta_{0}$. Also, due to strong fluctuations in ID systems, we expect that $\rho_{c}(T) / \rho_{c}(T$ $=0) \approx\left(\Delta / \Delta_{0}\right)^{2}$ is a reasonable approximation.

[9] D. Feinberg and J. Friedel, J. Phys. (Paris) 49, 485 (1988).

[10] J. S. Langer and M. E. Fisher, Phys. Rev. Lett. 19, 560 (1967).

[11] S. Ramakrishna (to be published).

[12] M. Peach and J. S. Koehler, Phys. Rev. 80, 436 (1950).

[13] S. J. Putterman, Superfluid Hydrodynamics (NorthHolland, Amsterdam, 1975).

[14] For $\mathrm{NbSe}_{3}, K_{z} \Delta_{0}^{2} \approx 5.6 \times 10^{-12} \mathrm{~J} / \mathrm{m}, K_{x} \Delta_{0}^{2}=6.2 \times 10^{-13}$ $\mathrm{J} / \mathrm{m}, \quad K_{y} \Delta_{0}^{2} \approx 2.24 \times 10^{-13} \mathrm{~J} / \mathrm{m}, \quad \rho_{s} \approx 10^{27} \mathrm{~m}^{-3}$, and $Q \approx 10^{10} \mathrm{~m}^{-1}$ from Ref. [1]. As described in Ref. [9], the effective elasticity for the nucleation of an edge loop is $\bar{K}=\left(K_{x} K_{v}\right)^{1 / 2}$.
[15] U. Eckern and S. Ramakrishna, Phys. Rev. B 44, 984 (1991). We note that the $\gamma$ used in this paper differs from that in the present one.

[16] Studying the equation of motion in the bulk (i.e., far from current contacts), we get

$$
\begin{aligned}
\gamma \dot{\phi}_{0}-F_{\text {imp }}\left(\phi_{0}\right)-K_{x} \Delta^{2} & \frac{\partial^{2} \phi_{0}}{\partial x^{2}} \\
& -K_{y} \Delta^{2} \frac{\partial^{2} \phi_{0}}{\partial y^{2}}-K_{z} \Delta^{2} \frac{\partial^{2} \phi_{0}}{\partial z^{2}}=\frac{e \rho_{s}}{Q} \mathscr{E},
\end{aligned}
$$

where $\mathscr{E}$ is the electric field applied in the bulk when current $I_{\text {CDW }}$ flows. The phases are not imposed at the boundaries of our sample: They are not set at a voltage contact, only at a current contact. In the bulk, the voltage developed (across a length $L$ ) when current $I_{C D W}$ flows is equal to $\mathscr{E}_{T} L+I_{\mathrm{CDw}} r_{\mathrm{CDw}} L$. This implies that the left-hand side of the above equation, when averaged over impurities, time, and space, equals $\left(e \rho_{s} / Q\right)\left[\mathscr{E}_{T}\right.$ $\left.+I_{\text {CDw }} r_{\text {CDW }}\right]$ when current $I_{\text {CDW flows. After averaging }}$ over the transverse dimensions, we include it in the text as shown.

[17] From high-velocity perturbation theory, the pinning energy falls off rapidly [11] $\left(-1 / E^{2}\right)$ for large fields. Hence, to a good approximation, above threshold the pinning energy density is zero.

[18] We would see interesting effects akin to the resistive transition in superconductors [J. S. Langer and V. Ambegaokar, Phys. Rev. 164, 498 (1967)] if the phase strain ( $k=\partial \phi / Q \partial z)$ corresponding to (8) were increased beyond $k_{c} \approx 1 / Q \xi_{A}^{l}$. This requires a $V_{\mathrm{ps}} \sim$ tens of $\mathrm{mV}$, much larger than achievable in the samples studied. Additionally, even for small $V_{\mathrm{ps}}, \phi_{\mathrm{I}}$ is a large phase deformation [see $\mathrm{H}$. Matsukawa and $\mathrm{H}$. Takayama, Physica (Amsterdam) 143B, 80 (1986)]. However, the pinning-related properties of the CDW will not change compared to the case of $\phi_{1}=0$ due to the random nature of the pinning [11]. We are hence justified in considering the case of small $\phi_{1}$.

[19] M. Tinkham, Introduction to Superconductivity (Krieger, Melbourne, FL, 1980), Chap. 7. 